\title{
Optimal operation of microgrids with risk-constrained state of charge
}

\author{
Jie Lei ${ }^{1}$, Christian A. Hans ${ }^{2}$ and Pantelis Sopasakis ${ }^{3}$
}

\begin{abstract}
In this paper we present a stochastic scenariobased model predictive control (MPC) approach for the operation of islanded microgrids with high share of renewable energy sources. We require that the stored energy remains within given bounds with a certain probability using risk-based constraints as convex approximations of chance constraints. We show that risk constraints can generally be cast as conic constraints and, unlike chance constraints, can control both the number and average magnitude of constraint violations. Lastly, we demonstrate the risk-constrained stochastic MPC in a numerical case study.
\end{abstract}

\section{INTRODUCTION}

The high share of renewable energy sources in islanded microgrids (MGs) exposes them to increased uncertainty due to the weather-dependent nature of energy production. The pressing need to minimize the use of conventional generators and maximize infeed from renewable sources while respecting constraints on stored energy and power has led to a wide adoption of model predictive control (MPC) [1]-[3]. MPC additionally allows to make use of feed-forward information using forecasting models (see, e.g., [4]) of wind speed, solar irradiance and load demand.

Early works on deterministic MPC [1], [5], [6] are giving way to methods that take the associated uncertainty into consideration. Worst-case approaches [7], [8] can prove overly conservative, especially in presence of higly uncertain wind, irradiance and load. Expectation-based (risk-neutral) stochastic MPC formulations have been proposed involving either (typically independent) processes with continuous distributions [9] or scenario-based formulations [2]. Recently, a multistage risk-averse approach was proposed in [3] to account for the fact that distributions are never known exactly.

In stochastic MPC formulations, constraints become stochastic too. These can be imposed for all realizations of uncertainty [2], [3]. A more appropriate and less conservative approach is to require that the probability of constraints violations is sufficiently small. Such chance-constrained formulations are popular, e.g., in optimal power flow problems [10]. Chance constraints are generally nonconvex. In certain cases, e.g., for certain continuous distributions and linear systems, the inverse cumulative is known and chance constraints can be simplified [9], [11]. The wellknow framework of Nemirovski and Shapiro [12] for convex approximations of chance constraints is widely known.

\footnotetext{
${ }^{1}$ Jie Lei is with State Key Laboratory of Power Transmission Equipment \& System Security and New Technology at Chongqing University, China, jlei02@qub.ac.uk.

${ }^{2}$ Christian A. Hans is with the Control Systems Group at Technische Universität Berlin, Germany, hans@control .tu-berlin. de.

${ }^{3}$ Pantelis Sopasakis is with the School of EEECS and the i-AMS Centre at Queen's University Belfast, UK, p.sopasakis@qub.ac.uk.
}

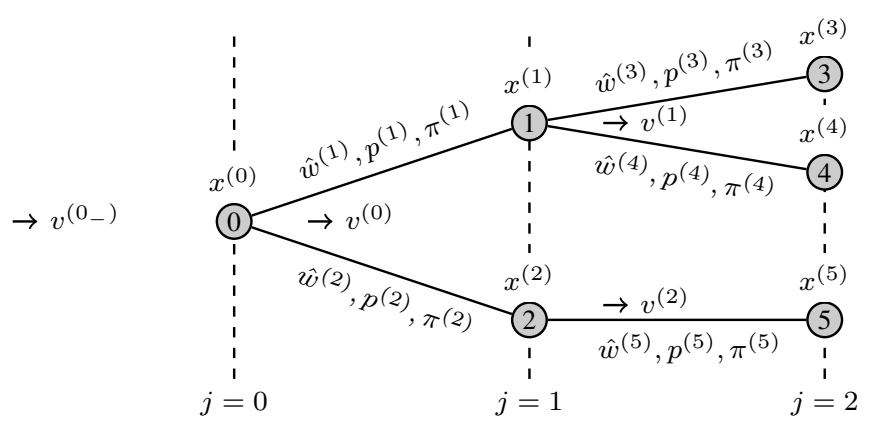

Fig. 1. Simple scenario tree from [16]. In this tree, $\operatorname{nodes}(0)=\{0\}$, $\operatorname{nodes}(1)=\{1,2\}$ and $\operatorname{nodes}(2)=\{3,4,5\}$. Moreover, the child nodes are child $(0)=\{1,2\}$, child $(1)=\{3,4\}$, child $(2)=\{5\}$ and consequently $\operatorname{anc}(3)=\operatorname{anc}(4)=1, \operatorname{anc}(5)=2, \operatorname{anc}(1)=\operatorname{anc}(2)=0$.

Some approximations have also been proposed such as the stochastic tubes approach of [13] for constraint tightening, and machine learning [14].

In this paper we propose a stochastic scenario-based MPC scheme for the operation of islanded microgrids with a high share of renewable sources. We formulate a stochastic MPC problem with chance constraints on the state of charge of storage units. Moreover, we employ the approach of [15] to overapproximate chance constraints by convex conic constraints using coherent risk measures. Lastly, we demonstrate the proposed chance-constrained MPC via realistic simulations with high renewable share using real-world irradiance and load data and time series forecast models.

Notation: Hereafter we denote by $\mathbb{N}, \mathbb{N}_{0}$ and $\mathbb{R}$ the sets of natural numbers, nonnegative integer and real numbers respectively. We denote the set of integers between $k$ and $k^{\prime}$ by $\mathbb{N}_{\left[k, k^{\prime}\right]}$. Let $\mathbb{R}_{>0}=\{x \in \mathbb{R} \mid x>0\}$. Likewise define $\mathbb{R}_{\geq 0}, \mathbb{R}_{\leq 0}$ and $\mathbb{R}_{<0}$. The Euclidean norm is $\|\cdot\|_{2}$. Given a set of indices $\mathbb{N}_{\left[k, k^{\prime}\right]},\left[x_{i}\right]_{i \in \mathbb{N}_{\left[k, k^{\prime}\right]}}$ is shorthand for $\left[x_{k}, \cdots, x_{k^{\prime}}\right]$.

\section{MICROGRID MODEL}

In what follows, some basics on scenario trees are introduced. Moreover, the relation of model variables is discussed and a detailed overview over the MG model is provided.

\section{A. Introduction on scenario trees}

In the context of this work, the probability distribution of load and available renewable infeed is assumed to be given in the form of a forecast scenario tree.

A scenario tree is formed of $N_{\mathrm{n}} \in \mathbb{N}$ nodes. Each node $i \in \mathbb{N}_{\left[0, N_{\mathrm{n}}-1\right]}$ is associated with prediction step $j \in \mathbb{N}_{[0, N]}$, also referred to as stage, denoted as stage $(i)$. All nodes associated with stage $j$ are collected in the set nodes $(j)$. The 
TABLE I

MODEL-SPECIFIC VARIABLES

\begin{tabular}{clcc}
\hline Symbol & Explanation & Unit & Size \\
\hline$x$ & Energy of storage units (state) & $\mathrm{pu} \mathrm{h}$ & $N_{\mathrm{s}}$ \\
\hline$u_{\mathrm{t}}$ & Control inputs of conventional units & $\mathrm{pu}$ & $N_{\mathrm{t}}$ \\
$u_{\mathrm{s}}$ & Control inputs of storage units & $\mathrm{pu}$ & $N_{\mathrm{s}}$ \\
$u_{\mathrm{r}}$ & Control inputs of renewable units & $\mathrm{pu}$ & $N_{\mathrm{r}}$ \\
$u$ & Control inputs of all units & $\mathrm{pu}$ & $N_{\mathrm{u}}$ \\
$\delta_{\mathrm{t}}$ & Boolean control inputs of conv. units & - & $N_{\mathrm{t}}$ \\
$v$ & Vector of all control inputs & - & $N_{\mathrm{v}}$ \\
\hline$w_{\mathrm{r}}$ & Uncertain available renewable power & $\mathrm{pu}$ & $N_{\mathrm{r}}$ \\
$w_{\mathrm{d}}$ & Uncertain load & $\mathrm{pu}$ & $N_{\mathrm{d}}$ \\
$w$ & Vector of all uncertain inputs & $\mathrm{pu}$ & $N_{\mathrm{w}}$ \\
\hline$p_{\mathrm{t}}$ & Power of conventional units & $\mathrm{pu}$ & $N_{\mathrm{t}}$ \\
$p_{\mathrm{s}}$ & Power of storage units & $\mathrm{pu}$ & $N_{\mathrm{s}}$ \\
$p_{\mathrm{r}}$ & Power of renewable units & $\mathrm{pu}$ & $N_{\mathrm{r}}$ \\
$p$ & Power of all units & $\mathrm{pu}$ & $N_{\mathrm{u}}$ \\
$p_{\mathrm{e}}$ & Power over transmission lines & $\mathrm{pu}$ & $N_{\mathrm{e}}$ \\
\hline & & &
\end{tabular}

number of nodes at stage $j$ is $n_{j}=|\operatorname{nodes}(j)|$. Node $i=0$ at stage $j=0$ is referred to as the root node, and the nodes at stage $N$, i.e., the elements of $\operatorname{nodes}(N)$, are referred to as leaf nodes. Each node $i \in \operatorname{nodes}(j)$ at stage $j \in \mathbb{N}_{[1, N]}$ is associated with an ancestor node $i_{-} \in \operatorname{nodes}(j-1)$ at stage $j-1$ which can be accessed via $i_{-}=\operatorname{anc}(i)$. All nodes at stage $j$ that share a common ancestor $i \in \operatorname{nodes}(j-1)$ are referred to as child nodes of $i$ and are collected in $\operatorname{child}(i) \subseteq \operatorname{nodes}(j)$. Each node $i$ is associated with a probability $\pi^{(i)} \in(0,1]$. For all stages $j \in \mathbb{N}_{[0, N]}$, it holds that $\sum_{i \in \operatorname{nodes}(j)} \pi^{(i)}=1$. The probability of node $i \in \mathbb{N}_{\left[0, N_{\mathrm{n}}-1\right]} \backslash \operatorname{nodes}(N)$ is linked to the probabilities of its children via $\pi^{(i)}=\sum_{i_{+} \in \operatorname{child}(i)} \pi^{\left(i_{+}\right)}$. An example of a scenario tree is shown in Fig. 1 .

\section{B. Generic microgrid model}

We consider an MG that comprises an arbitrary finite number of conventional and renewable generators, storage units and loads. These components are connected to each other via AC transmission lines. With the variables summarized in Table I. the behavior of the MG is modeled for all nodes $i_{+} \in \mathbb{N}_{\left[1, N_{\mathrm{n}}-1\right]}$ with $i=\operatorname{anc}\left(i_{+}\right)$by

$$
\begin{aligned}
x^{\left(i_{+}\right)} & =A x^{(i)}+B p^{\left(i_{+}\right)}, \\
h_{x} & \leq H_{x} x^{\left(i_{+}\right)}, \\
p^{\left(i_{+}\right)} & =f_{p}\left(v^{(i)}, w^{\left(i_{+}\right)}\right), \\
h_{v p} & \leq H_{v p}\left[v^{(i)^{\top}} \quad p^{\left(i_{+}\right)^{\top}}\right]^{\top} .
\end{aligned}
$$

Here, $x^{(i)}$ represents the state, i.e., the energy stored in storage units at node $i$. Moreover, $u^{(i)}=\left[\begin{array}{lll}u_{\mathrm{t}}^{(i)^{\top}} & u_{\mathrm{s}}^{(i)^{\top}} & u_{\mathrm{r}}^{(i)^{\top}}\end{array}\right]^{\top}$ is a vector of real-valued control inputs and $\delta_{\mathrm{t}}^{(i)} \in\{0,1\}^{N_{\mathrm{t}}}$ a vector of Boolean inputs that indicates whether conventional unit $l \in \mathbb{N}_{\left[1, N_{\mathrm{t}}\right]}$ is enabled $\left(\delta_{\mathrm{t}, l}^{(i)}=1\right)$ or disabled $\left(\delta_{\mathrm{t}, l}^{(i)}=0\right)$. The control inputs are collected in $v^{(i)}=\left[\begin{array}{ll}u^{(i)^{\top}} & \delta^{(i)^{\top}}\end{array}\right]^{\top}$. Finally, the uncertain input at node $i_{+} \in \operatorname{child}(i)$ is $w^{\left(i_{+}\right)}=\left[w_{\mathrm{r}}^{\left(i_{+}\right)^{\top}} \quad w_{\mathrm{d}}^{\left(i_{+}\right)^{\top}}\right]^{\top}$ and the units'

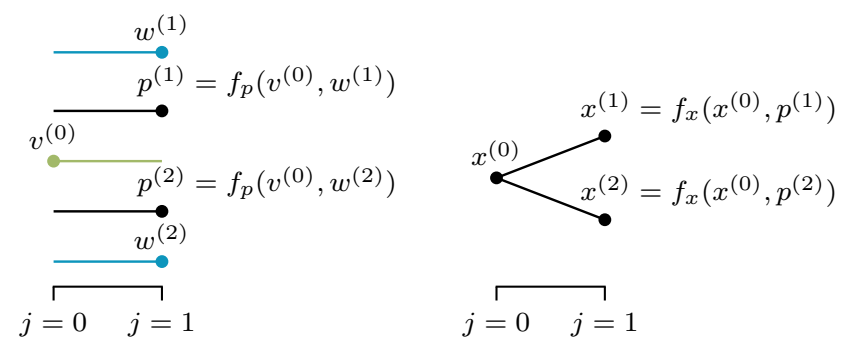

Fig. 2. Relation of variables in scenario tree. Source: [16].

power is $p^{\left(i_{+}\right)}=\left[p_{\mathrm{t}}^{\left(i_{+}\right)^{\top}} \quad p_{\mathrm{s}}^{\left(i_{+}\right)^{\top}} \quad p_{\mathrm{r}}^{\left(i_{+}\right)^{\top}}\right]^{\top}$.

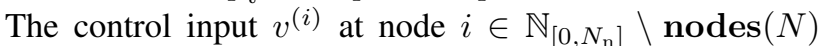
is present between time instants $j=\operatorname{stage}(i)$ and $j+1$. The uncertain input $w^{\left(i_{+}\right)}$and the power $p^{\left(i_{+}\right)}$at nodes $i_{+} \in \operatorname{child}(i)$ are associated with the same time interval. The reason for this is that power and uncertain input are associated with the state $x^{\left(i_{+}\right)}$that they result in.

An example of this relation is illustrated in Fig. 2. Here, the control input between instants $j=0$ and $j=1$ is $v^{(0)}$. In the example, two realizations of the uncertain input are predicted, $w^{(1)}$ and $w^{(2)}$. Thus, for the same control input $v^{(0)}$, different values of load and available renewable infeed can occur. The power of the units, which is collected in $p^{(1)}$ and $p^{(2)}$, changes with the control input and the uncertain input. Similarly, the state changes with these power values. As shown in Fig. 2, $x^{(1)}$ is a function of $x^{(0)}$ and $p^{(1)}$ and $x^{(2)}$ a function of $x^{(0)}$ and $p^{(2)}$.

Note that by introducing additional free variables, (1c) can be transformed into a set of affine equality and inequality constraints (see also Section II-D and [3]). Thus, (1) only comprises affine constraints and can therefore be used to formulate mixed-integer quadratic programs.

In what follows, we will discuss the equations used to formulate the generic model (1). We start with the dynamics.

\section{Dynamics}

The dynamics of the stored energy $x^{\left(i_{+}\right)}$are modeled using a discrete-time state model. With $A=I_{N_{\mathrm{s}}}$ and $B=\left[\begin{array}{lll}0_{N_{\mathrm{s}} \times N_{\mathrm{t}}} & -T_{\mathrm{s}} I_{N_{\mathrm{s}}} & 0_{N_{\mathrm{s}} \times N_{\mathrm{r}}}\end{array}\right]$ the model reads

$$
x^{\left(i_{+}\right)}=A x^{(i)}+B p^{\left(i_{+}\right)} \text {with } \quad x(0)=x_{0}
$$

and $i=\operatorname{anc}\left(i_{+}\right)$. This is precisely Equation (1a).

\section{Energy-related limits}

The stored energy at all nodes $i_{+} \in \mathbb{N}_{\left[1, N_{\mathrm{n}}\right]}$ is limited by

$$
x^{\min } \leq x^{\left(i_{+}\right)} \leq x^{\max }
$$

with $x^{\min } \in \mathbb{R}_{\geq 0}^{N_{\mathrm{s}}}$ and $x^{\max } \in \mathbb{R}_{\geq 0}^{N_{\mathrm{s}}}$. From these inequalities, (1b) can be easily deduced.

\section{E. Power of units}

The units' power is not only affected by the power setpoints but also by the uncertain load and available renewable infeed. This is taken into account by considering different realisations of the uncertain input $w^{\left(i_{+}\right)}$for each control 
input $v^{(i)}$. The effects of the control input and the uncertain input on the power enter the generic model (1) via function $f_{p}$ which is implicitly defined by constraints (4)-6).

1) Transmission network: A power equilibrium of generation, consumption and storage power must be ensured at all times. This can be modelled by the equality constraint

$$
1_{N_{\mathrm{t}}}^{\top} p_{\mathrm{t}}^{\left(i_{+}\right)}+1_{N_{\mathrm{s}}}^{\top} p_{\mathrm{s}}^{\left(i_{+}\right)}+1_{N_{\mathrm{r}}}^{\top} p_{\mathrm{r}}^{\left(i_{+}\right)}+1_{N_{\mathrm{d}}}^{\top} w_{\mathrm{d}}^{\left(i_{+}\right)}=0
$$

2) Renewable units: The power of the renewable units $p_{\mathrm{r}}^{\left(i_{+}\right)}$cannot exceed the weather-dependent available renewable infeed $w_{\mathrm{r}}^{\left(i_{+}\right)}$. The control input $u_{\mathrm{r}}^{(i)}$ allows to limit $p_{\mathrm{r}}^{\left(i_{+}\right)}$below $w_{\mathrm{r}}^{\left(i_{+}\right)}$. If the available renewable power $w_{\mathrm{r}, l}^{\left(i_{+}\right)}$ of unit $l \in \mathbb{N}_{\left[1, N_{\mathrm{r}}\right]}$ is below the power setpoint $u_{\mathrm{r}, l}^{(i)}$, then the infeed of this unit equals the available renewable power. If the available renewable power $w_{\mathrm{r}, l}^{\left(i_{+}\right)}$is above the power setpoint $u_{\mathrm{r}, l}^{(i)}$, then the infeed of this unit equals the power setpoint. This can be modelled via

$$
p_{\mathrm{r}}^{\left(i_{+}\right)}=\min \left(u_{\mathrm{r}}^{(i)}, w_{\mathrm{r}}^{\left(i_{+}\right)}\right)
$$

3) Power sharing of grid-forming units: The lower control layers of the MG are assumed to be designed such that fluctuations of load and renewable infeed are distributed among grid-forming storage and conventional units in a proportional manner. This ensures that (4) holds in presence of uncertain load and renewable infeed. It can be implemented using, for example, decentralized droop control [17]. Typically, power sharing is implemented on a much faster time scale than operation control. Nevertheless, power sharing needs to be considered in the constraints as it links the power of the conventional and storage units to the fluctuations of the uncertain input. Using the additional variable $\mu^{\left(i_{+}\right)} \in \mathbb{R}$, it can be included in the MPC formulation by [3]

$$
\begin{aligned}
& K_{\mathrm{s}}\left(p_{\mathrm{s}}^{\left(i_{+}\right)}-u_{\mathrm{s}}^{(i)}\right)=\mu^{\left(i_{+}\right)} \\
& K_{\mathrm{t}}\left(p_{\mathrm{t}}^{\left(i_{+}\right)}-u_{\mathrm{t}}^{(i)}\right)=\mu^{\left(i_{+}\right)} \delta_{\mathrm{t}}^{(i)} .
\end{aligned}
$$

As indicated in [3], (4)-(6) can be equivalently expressed by affine equality and inequality constraints using additional decision variables and the so-called Big-M method (see, e.g., [18]). Consequently, $f_{p}\left(v^{(i)}, w^{\left(i_{+}\right)}\right)$in (1c) can be used to formulate mixed-integer optimization problems.

\section{F. Power-related limits}

The limits on power and power setpoints can be divided into limits on units and limits on transmission lines. In detail, (1d) is composed of the following inequalities.

1) Renewable units: Power and control input are limited by $p_{\mathrm{r}}^{\min } \in \mathbb{R}_{\geq 0}^{N_{\mathrm{r}}}$ and $p_{\mathrm{r}}^{\max } \in \mathbb{R}_{\geq 0}^{N_{\mathrm{r}}}$, i.e.,

$$
\begin{aligned}
& p_{\mathrm{r}}^{\min } \leq p_{\mathrm{r}}^{\left(i_{+}\right)} \leq p_{\mathrm{r}}^{\max } \\
& p_{\mathrm{r}}^{\min } \leq u_{\mathrm{r}}^{(i)} \leq p_{\mathrm{r}}^{\max }
\end{aligned}
$$

2) Conventional units: If a unit is disabled, then power and setpoint are zero, otherwise power and setpoint are limited by a minimum and a maximum value. With $p_{\mathrm{t}}^{\min } \in \mathbb{R}_{\geq 0}^{N_{\mathrm{t}}}$ and $p_{\mathrm{t}}^{\max } \in \mathbb{R}_{\geq 0}^{N_{\mathrm{t}}}$, this can be expressed by

$$
\begin{aligned}
& \operatorname{diag}\left(p_{\mathrm{t}}^{\min }\right) \delta_{\mathrm{t}}^{(i)} \leq p_{\mathrm{t}}^{\left(i_{+}\right)} \leq \operatorname{diag}\left(p_{\mathrm{t}}^{\max }\right) \delta_{\mathrm{t}}^{(i)}, \\
& \operatorname{diag}\left(p_{\mathrm{t}}^{\min }\right) \delta_{\mathrm{t}}^{(i)} \leq u_{\mathrm{t}}^{(i)} \leq \operatorname{diag}\left(p_{\mathrm{t}}^{\max }\right) \delta_{\mathrm{t}}^{(i)} .
\end{aligned}
$$

3) Storage units: The power and the power setpoints are limited by $p_{\mathrm{s}}^{\min } \in \mathbb{R}_{\leq 0}^{N_{\mathrm{s}}}$ and $p_{\mathrm{s}}^{\max } \in \mathbb{R}_{\geq 0}^{N_{\mathrm{s}}}$, i.e.,

$$
\begin{aligned}
& p_{\mathrm{s}}^{\min } \leq p_{\mathrm{s}}^{\left(i_{+}\right)} \leq p_{\mathrm{s}}^{\max }, \\
& p_{\mathrm{s}}^{\min } \leq u_{\mathrm{s}}^{(i)} \leq p_{\mathrm{s}}^{\max } .
\end{aligned}
$$

4) Transmission network: The transmission network is included using the linear DC power flow approximations for AC grids (see, e.g. [16]). As the power equilibrium is already implemented via (4), we can directly deduce the power flowing over the transmission lines $p_{\mathrm{e}}^{\left(i_{+}\right)}$via

$$
p_{\mathrm{e}}^{\left(i_{+}\right)}=F\left[\begin{array}{llll}
p_{\mathrm{t}}^{\left(i_{+}\right)} & p_{\mathrm{s}}^{\left(i_{+}\right)} & p_{\mathrm{r}}^{\left(i_{+}\right)} & w_{\mathrm{d}}^{\left(i_{+}\right)}
\end{array}\right]^{\top}
$$

with $F \in \mathbb{R}^{N_{\mathrm{e}} \times\left(N_{\mathrm{u}}+N_{\mathrm{d}}\right)}$. Naturally, $p_{\mathrm{e}}^{\left(i_{+}\right)}$is bounded, i.e.,

$$
p_{\mathrm{e}}^{\min } \leq F\left[p_{\mathrm{t}}^{\left(i_{+}\right)} \quad p_{\mathrm{s}}^{\left(i_{+}\right)} \quad p_{\mathrm{r}}^{\left(i_{+}\right)} \quad w_{\mathrm{d}}^{\left(i_{+}\right)}\right]^{\top} \leq p_{\mathrm{e}}^{\max }
$$

with $p_{\mathrm{e}}^{\min } \in \mathbb{R}_{\leq 0}^{N_{\mathrm{e}}}$ and $p_{\mathrm{e}}^{\max } \in \mathbb{R}_{\geq 0}^{N_{\mathrm{e}}}$.

This completes the introduction of the control-oriented MG model. Based on this section, we will now formulate a cost function for islanded MG with high renewable share.

\section{OpERATING COSTS}

The operating cost of the microgrid is motivated by [2]. It is composed of (i) fuels costs of the conventional units, $\ell_{\mathrm{t}}^{\mathrm{f}}$, (ii) switching costs of the conventional units, $\ell_{\mathrm{t}}^{\mathrm{s}}$, and (iii) costs incurred by limiting potential renewable infeed, $\ell_{\mathrm{r}}$. For scenario trees of the form described in Section III, the cost associated with node $i_{+} \in \mathbb{N}_{\left[1, N_{\mathrm{n}}\right]}$ is

$$
\begin{aligned}
\ell\left(v^{(i)}, v^{\left(i_{-}\right)}, p^{\left(i_{+}\right)}\right)=\left(\ell_{\mathrm{t}}^{\mathrm{f}}\left(v^{(i)}, p^{\left(i_{+}\right)}\right)+\right. \\
\left.\quad \ell_{\mathrm{t}}^{\mathrm{s}}\left(v^{(i)}, v^{\left(i_{-}\right)}\right)+\ell_{\mathrm{r}}\left(p^{\left(i_{+}\right)}\right)\right) \gamma^{\text {stage }\left(i_{+}\right)},
\end{aligned}
$$

with $i=\operatorname{anc}\left(i_{+}\right)$and $i_{-}=\operatorname{anc}(i)$. Here, discount factor $\gamma \in(0,1]$ is used to put an emphasis on near future decisions.

In (11), decision variables associated with different nodes are used. The reason for this is that the power $p^{\left(i_{+}\right)}$depends on control input $v^{(i)}, i=\operatorname{anc}\left(i_{+}\right)$. In addition, the switching costs associated with $v^{(i)}$ depend on the Boolean input $\delta_{\mathrm{t}}^{\left(i_{-}\right)}$ at the previous time instant which is part of $v^{\left(i_{-}\right)}$.

The fuel cost of conventional units is approximately [19]

$$
\ell_{\mathrm{t}}^{\mathrm{f}}\left(v^{(i)}, p^{\left(i_{+}\right)}\right)=c_{\mathrm{t}}^{\top} \delta_{\mathrm{t}}^{(i)}+c_{\mathrm{t}}^{\prime^{\top}} p_{\mathrm{t}}^{\left(i_{+}\right)}+\left\|\operatorname{diag}\left(c_{\mathrm{t}}^{\prime \prime}\right) p_{t}^{\left(i_{+}\right)}\right\|_{2}^{2}
$$

with weights $c_{\mathrm{t}} \in \mathbb{R}_{>0}^{T}, c_{\mathrm{t}}^{\prime} \in \mathbb{R}_{>0}^{T}$, and $c_{\mathrm{t}}^{\prime \prime} \in \mathbb{R}_{>0}^{T}$. Moreover, the cost incurred by switching the conventional generators is

$$
\ell_{\mathrm{t}}^{\mathrm{S}}\left(v^{(i)}, v^{\left(i_{-}\right)}\right)=\left\|\operatorname{diag}\left(c_{\mathrm{t}}^{\mathrm{s}}\right)\left(\delta_{\mathrm{t}}^{\left(i_{-}\right)}-\delta_{\mathrm{t}}^{(i)}\right)\right\|_{2}^{2}
$$

with weight $c_{\mathrm{t}}^{\mathrm{s}} \in \mathbb{R}_{>0}^{T}$. For nodes $i_{+} \in \mathbb{N}_{\left[1, N_{\mathrm{n}}-1\right]}$ with $\operatorname{anc}\left(i_{+}\right)=0$, $12 \mathrm{~b}$ becomes $\ell_{\mathrm{t}}^{\mathrm{s}}\left(v^{(0)}, v^{\left(0_{-}\right)}\right)$. Here, $v^{\left(0_{-}\right)}$is the input applied at the previous execution of the controller. 
The goal in the operation of renewable units is to use as much weather-dependent available power as possible. Using $p_{\mathrm{r}}^{\max }$ from Section II and weight $c_{\mathrm{r}} \in \mathbb{R}_{>0}^{R}$, this can be encoded into the cost function via

$$
\ell_{\mathrm{r}}\left(p^{\left(i_{+}\right)}\right)=\left\|\operatorname{diag}\left(c_{\mathrm{r}}\right)\left(p_{\mathrm{r}}^{\max }-p_{\mathrm{r}}^{\left(i_{+}\right)}\right)\right\|_{2}^{2}
$$

In favour of a more compact notation, we introduce the cost variable $Z^{\left(i_{+}\right)}$for every node $i_{+} \in \mathbb{N}_{\left[1, N_{\mathrm{n}}-1\right]}$, i.e., $Z^{\left(i_{+}\right)}=\ell\left(v^{(i)}, v^{\left(i_{-}\right)}, p^{\left(i_{+}\right)}\right)$. The cost values at stage $j$ can be collected in $Z_{j}=\left[Z^{\left(i_{+}\right)}\right]_{i_{+} \in \operatorname{nodes}(j)}$, which defines a random variable on the probability space nodes $(j)$. Using the probabilities of stage $j, \pi_{j}=\left[\pi^{\left(i_{+}\right)}\right]_{i_{+} \in \operatorname{nodes}(j)}$, the expected cost at stage $j$ is

$$
\mathbf{E}^{\pi_{j}}\left(Z_{j}\right)=\pi_{j}^{\top} Z_{j}
$$

The total cost along the prediction horizon, $Z \in \mathbb{R}$, is the sum of expected costs over all stages of the tree, i.e.,

$$
Z=\sum_{j=1}^{N} \mathbf{E} \pi_{j}\left(Z_{j}\right)=\sum_{i_{+}=1}^{N_{\mathrm{n}}-1} \pi^{\left(i_{+}\right)} Z^{\left(i_{+}\right)} .
$$

Using the cost and the MG model from Section II. we can now formulate a chance-constrained MPC problem for islanded MGs.

\section{RISK-CONSTRAINED MODEL PREDICTIVE CONTROL}

In this section we shall state the chance-constrained and risk-constrained MPC problems.

\section{A. Chance-constrained optimal control formulations}

Consider the following stochastic optimal control problem with horizon $N$

$$
\underset{\boldsymbol{v}, \boldsymbol{x}, \boldsymbol{p}}{\operatorname{Minimize}} Z
$$

subject to the system dynamics and constraints in Equations (1) for all $i_{+} \in \mathbb{N}_{\left[1, N_{\mathrm{n}}-1\right]}$ with $i=\operatorname{anc}\left(i_{+}\right)$, which consists in minimizing the expected value of the cost defined in (15). The minimization is carried out over $\boldsymbol{x}=\left[x^{(i)}\right]_{i \in \mathbb{N}_{\left[0, N_{\mathrm{n}}-1\right]}}$, $\boldsymbol{v}=\left[v^{(i)}\right]_{i \in \mathbb{N}_{\left[0, N_{\mathrm{n}}-1\right]} \backslash \operatorname{nodes}(N)}$ and $\boldsymbol{p}=\left[p^{(i)}\right]_{i \in \mathbb{N}_{\left[1, N_{\mathrm{n}}-1\right]}}$ and given that the state at the root node, $x^{(0)}$, is equal to the measured state. We need to impose that the state of charge of each storage unit $s \in \mathbb{N}_{\left[1, N_{\mathrm{s}}\right]}$ at each stage $j$, $x_{s, j}=\left[x_{s}^{(i)}\right]_{i \in \operatorname{nodes}(j)}$, which is a random variable, remains bounded between $\tilde{x}^{\min } \geq x^{\min }$ and $\tilde{x}^{\max } \leq x^{\max }$ in a probabilistic sense. These tighter bounds serve to reduce the range of the state of charge which typically comes with positive effect on the aging of storage units (see, e.g., [20]).

To require that $x_{s, j}$ satisfies the constraints for all possible realizations of the uncertain renewable infeed and load can be overly conservative. A possible alternative is to require that the probability of constraint satisfaction at every storage unit $s$ is adequately high, i.e.,

$$
\mathrm{P}_{j}\left[x_{s, j} \notin\left[\tilde{x}^{\min }, \tilde{x}^{\max }\right]\right] \leq \alpha,
$$

for all stages $j \in \mathbb{N}_{[1, N]}$, for some $\alpha \in[0,1]$, where $\mathrm{P}_{j}$ is the probability measure of $\operatorname{nodes}(j)$ associated with probability vector $\pi_{j}$. This condition can be rewritten using the valueat-risk operator of a random variable $X$ on the probability space $\operatorname{nodes}(j)$,

$$
\mathrm{V} @ \mathrm{R}_{\alpha}[X]:=\inf \left\{t: \mathrm{P}_{j}[X>t] \leq \alpha\right\}
$$

In fact, the above probabilistic constraints are equivalent to

$$
\mathrm{V} @ \mathrm{R}_{\alpha}\left[d_{j}\left(x_{s, j}\right)\right] \leq 0,
$$

for all $j \in \mathbb{N}_{[1, N]}$, where $d_{j}$ is the distance-to-set function defined as $d_{j}(x)=\min _{y \in\left[\tilde{x}_{s}^{\min }, \tilde{x}_{s}^{\max }\right]}|x-y|$. Onesided constraints of the form $\mathrm{P}_{j}\left[x_{s, j}>\tilde{x}^{\max }\right] \leq \alpha$, can be imposed by considering functions of the form $d_{j}^{\max }(x)=\min _{y \leq \tilde{x}_{s}^{\max }}|x-y|$.

Such probabilistic constraints are nonconvex, call for additional binary variables and can lead to computationally intractable optimization problems, while they limit the frequency of violations, but not their magnitude. Indeed, in order to impose the constraint of 16 we define the binary variable $\tau_{s}^{(i)} \in\{0,1\}^{n_{j}}$ which is such that

$$
\tau_{s}^{(i)}=0 \Longleftrightarrow d_{j}\left(x_{s}^{(i)}\right) \leq 0,
$$

for all $i \in \operatorname{nodes}(j)$. Using a standard big-M relaxation, we choose $M \geq \max \left(x^{\max }-x^{\min }\right), m \leq 0$ and a small tolerance $\epsilon>0$ to rewrite (19) as

$$
\epsilon+(m-\epsilon) \tau_{j}^{(i)} \leq d_{j} \leq M\left(1-\tau_{j}^{(i)}\right),
$$

for all $i \in \operatorname{nodes}(j)$. Then, for each storage unit $s$,

$$
\mathrm{P}_{j}\left[d_{j}\left(x_{s}^{(i)}\right)>0\right] \leq \alpha \Longleftrightarrow \sum_{i \in \operatorname{nodes}(j)} \pi^{(i)} \tau_{s}^{(i)} \geq 1-\alpha,
$$

which, by defining $\tau_{s, j}=\left[\tau_{s}^{(i)}\right]_{i \in \operatorname{nodes}(j)}$, can be equivalently written as $\pi_{j}^{\top} \tau_{s, j} \geq 1-\alpha$. In summary, the above chance constraints are equivalent to

$$
\begin{array}{r}
\tau_{s, j}^{(i)} \in\{0,1\}, \quad d_{j}\left(x_{s, j}^{(i)}\right) \leq \xi_{s}^{(i)}, \quad \pi_{j}^{\top} \tau_{s, j} \geq 1-\alpha, \\
\epsilon+(m-\epsilon) \tau_{s, j}^{(i)} \leq \xi_{s}^{(i)} \leq M\left(1-\tau_{j}^{(i)}\right),
\end{array}
$$

for $i \in \operatorname{nodes}(j)$, where we have introduced a relaxation of $d_{j}\left(x_{s, j}^{(i)}\right)$ by introducing the auxiliary variables $\xi_{s}^{(i)}$. Note that in order to impose chance constraints we need to introduce as many binary variables as the nodes of the tree times the number of storage units. Overall, the chance-constrained optimal control problem reads

$$
\underset{\boldsymbol{v}, \boldsymbol{x}, \boldsymbol{p}, \boldsymbol{\xi}, \boldsymbol{\tau}}{\operatorname{Minimize}} Z
$$

subject to the system dynamics and constraints in (1) and 22 for all $i_{+} \in \mathbb{N}_{\left[1, N_{\mathrm{n}}-1\right]}$ with $i=\operatorname{anc}\left(i_{+}\right)$. Note that the minimization is taken over $\boldsymbol{v}, \boldsymbol{x}, \boldsymbol{p}$

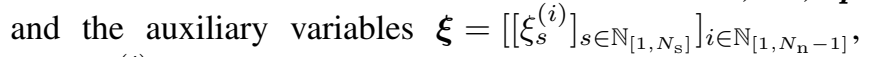

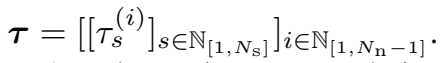

An alternative approach is to use risk-based constraints, which can control both the occurrence and the magnitude of violations. 


\section{B. Risk measures}

A risk measure is an operator that maps random variables to characteristic values. The risk of a random cost represents an "equivalent" fixed cost value. The expectation and maximum operators are examples of risk measures.

A risk measure $\rho$ on a finite probability space $(\Omega, \mathrm{P})$, with $\Omega=\{i\}_{i=1}^{n}$, is considered to be well-behaving if it is (i) convex, that is, for all random variables $X_{1}, X_{2}$ and $\lambda \in$ $[0,1]$, it is $\rho\left(\lambda X_{1}+(1-\lambda) X_{2}\right) \leq \lambda \rho\left(X_{1}\right)+(1-\lambda) \rho\left(X_{2}\right)$, (ii) monotone, in the sense that $\rho\left(X_{1}\right) \leq \rho\left(X_{2}\right)$ whenever $\mathrm{P}\left[X_{1}>X_{2}\right]=0$, (iii) translation equi-variant, i.e., $\rho(X+$ $c)=c+\rho(X)$ for all constants $c \in \mathbb{R}$, and (iv) positive homogeneous, that is $\rho(a X)=a \rho(X)$, for all $a \geq 0$. Risk measures that satisfy these requirements are called coherent and can be represented as

$$
\rho[X]=\sup _{\mu \in \mathcal{A}} \mathbf{E}^{\mu}[X]
$$

where $\mathbf{E}^{\mu}$ is the expectation operator with respect to a probability $\mu$ and $\mathcal{A}$ is a convex set of probabilities called the ambiguity set of $\rho$ [21, Theorem 6.5].

A widely used coherent risk measure is the average valueat-risk at level $\alpha$ denoted by $\mathrm{AV} @ \mathrm{R}_{\alpha}$; its ambiguity set over a finite, $n$-dimensional, probability space with probability vector $\pi \in \mathbb{R}^{n}$, is the polytope

$$
\mathcal{A}_{\alpha}=\left\{\mu \in \mathbb{R}^{n}: \sum_{i=1}^{n} \mu_{i}=1,0 \leq \alpha \mu \leq \pi\right\} .
$$

Note that $\mathcal{A}_{0}$ coincides with the whole probability simplex and $\mathrm{AV} @ \mathrm{R}_{0}[X]=\max _{i=1, \ldots, n}\left\{X_{i}: \pi_{i} \neq 0\right\}$, while $\mathcal{A}_{1}=$ $\{\pi\}$, therefore $\mathrm{AV} @ \mathrm{R}_{1}[X]=\mathbf{E}^{\mathrm{P}}[X]$.

A noteworthy property of the average value-at-risk is that it is a tight convex overapproximation of the value-at-risk, that is $\mathrm{AV} @ \mathrm{R}_{\alpha}[X] \geq \mathrm{V} @ \mathrm{R}_{\alpha}[X]$, therefore the probabilistic constraints of (18) are satisfied if

$$
\mathrm{AV} @ \mathrm{R}_{\alpha}\left[d_{j}\left(x_{s, j}\right)\right] \leq 0,
$$

for all $j \in \mathbb{N}_{[1, N]}$. Unlike V@R-based constraints, AV@Rbased constraints are convex and do not lead to overly cumbersome optimization problems. For given $\alpha \in[0,1]$, any

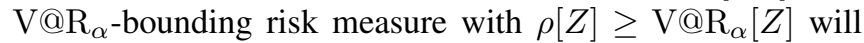
imply the satisfaction of the original probabilistic constraints.

The entropic value-at-risk, $\mathrm{EV} @ \mathrm{R}_{\alpha}$, is another example of such a risk measure [22]; its ambiguity set over a finite, $n$-dimensional, probability space with probability vector $\pi$ is the set of probability vectors, $\mu \in \mathbb{R}^{n}$, such that $\mathrm{D}_{\mathrm{KL}}(\mu \| \pi) \leq-\ln \alpha$, where $\mathrm{D}_{\mathrm{KL}}$ is the Kullback-Leibler divergence.

Lastly, the level of risk aversion can be determined from available data using statistical methods [23].

\section{Risk constraints}

Given a coherent $\mathrm{V} @ \mathrm{R}$-bounding risk measure $\rho_{j}$ defined on the probability space nodes $(j)$, which is equipped with the probability vector $\pi_{j}$, probabilistic constraints of the form given in (18) can be overapproximated the convex constraints

$$
\rho_{j}\left[C_{j}\right] \leq 0
$$

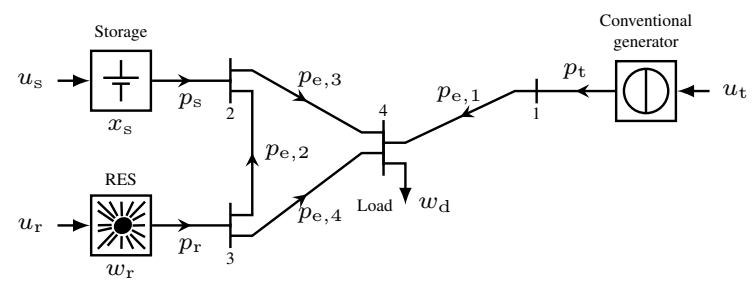

Fig. 3. Simple microgrid with storage unit, renewable and conventional generator as well as load. Source: [3].

where $C_{j}=d_{j}\left(x_{j}\right)$, for $j \in \mathbb{N}_{[1, N]}$. In this section we have dropped the index $s$ (cf. Equation 25) for the sake of simplicity. The associated risk-constrained optimal control problem is

$$
\underset{\boldsymbol{v}, \boldsymbol{x}, \boldsymbol{p}}{\operatorname{Minimize}} Z
$$

subject to 26 for all $j \in \mathbb{N}_{[1, N]}$ and the system dynamics and constraints in (1) for all $i_{+} \in \mathbb{N}_{\left[1, N_{n}-1\right]}$ with $i=\operatorname{anc}\left(i_{+}\right)$.

Since $\rho_{j}$ is a coherent risk measure, there is a closed convex set $\mathcal{A}_{\alpha, j}$ such that

$$
\rho_{j}\left[C_{j}\right]=\max _{\mu \in \mathcal{A}_{\alpha, j}} \mathbf{E}^{\mu}\left[C_{j}\right]=\max _{\mu \in \mathcal{A}_{\alpha, j}} \mu^{\top} C_{j} .
$$

The ambiguity set $\mathcal{A}_{\alpha, j}$ can generally be written in a conic form as follows

$$
\mathcal{A}_{\alpha, j}=\left\{\begin{array}{l|l}
\mu \in \mathbb{R}^{n_{j}} & \begin{array}{l}
\exists \nu \in \mathbb{R}^{r_{j}} \text { such that } \\
b_{\alpha, j}-E_{\alpha, j} \mu-F_{\alpha, j} \nu \in \mathcal{K}_{\alpha, j}
\end{array}
\end{array}\right\},
$$

where $b_{\alpha, j}, E_{\alpha, j}$ and $F_{\alpha, j}$ have appropriate dimensions, $r_{j} \in \mathbb{N}_{0}, \mathcal{K}_{\alpha, j}$ is a cone and it is implied that $\mathcal{A}_{\alpha, j}$ is a subset of the probability simplex of $\mathbb{R}^{n_{j}}$ [15].

For example, for $\mathrm{AV} @ \mathrm{R}_{\alpha}$ it is $E_{\alpha, j}=\left[\begin{array}{lll}I_{n_{j}} & -I_{n_{j}} & 1_{n_{j}}\end{array}\right]^{\top}$, $r_{j}=0, b_{\alpha, j}=\left[\begin{array}{lll}\alpha^{-1} \pi_{j}^{\top} & 0 & 1\end{array}\right]^{\top}$ and $\mathcal{K}_{\alpha, j}=\mathbb{R}^{2 n_{j}} \times\{0\}$. From convex duality, if there exist $\mu^{*} \in \mathbb{R}^{n_{j}}$ and $\nu^{*} \in \mathbb{R}^{r_{j}}$ so that $b-E \mu^{*}-F \nu^{*}$ is in the relative interior of $\mathcal{K}_{j}$, which is a very weak assumption, then $\rho_{j}\left[C_{j}\right]$, for $C_{j} \in \mathbb{R}^{n_{j}}$ can be written as

$$
\rho_{j}\left[C_{j}\right]=\min _{y}\left\{\begin{array}{l|l}
y^{\top} b_{\alpha, j} & \begin{array}{l}
E_{\alpha, j}^{\top} y=C_{j}, F_{\alpha, j}^{\top} y=0, \\
y \in \mathcal{K}_{\alpha, j}^{*}
\end{array}
\end{array}\right\},
$$

where $\mathcal{K}_{j}^{*}$ is the convex dual of $\mathcal{K}_{j}$ [24, Thm 2.4.1]. By virtue of 29), constraints 26) are equivalent to the existence of a $y_{j} \in \mathcal{K}_{\alpha, j}^{*}$ such that $E_{\alpha, j}^{\top} y_{j}=C_{j}$ and $F_{\alpha, j}^{\top} y_{j}=0$. This makes Problem $(\mathrm{P})$ into a mixed-integer conic optimization problem, yet without additional binary variables as it was the case with chance constraints.

\section{Simulations}

In the closed-loop simulations, the grid in Fig. 3 was considered. It is composed of a photovoltaic (PV) power plant, a conventional generator, and a storage unit, i.e., all basic components from Section [II are included. The units are connected to each other and to a load via transmission lines that all have a rated power of $1.3 \mathrm{pu}$. For all transmission lines a susceptance of $-20 \mathrm{pu}$ was considered. The remaining 
TABLE II

UNIT PARAMETERS AND WEIGHTS OF COST FUNCTION.

\begin{tabular}{|c|c|c|c|}
\hline Parameter & Value & Weight & Value \\
\hline$\left[p_{\mathrm{t}}^{\min }, p_{\mathrm{r}}^{\min }, p_{\mathrm{s}}^{\min }\right]$ & {$[0.4,0,-1] \mathrm{pu}$} & $c_{\mathrm{t}}$ & 0.1178 \\
\hline$\left[p_{\mathrm{t}}^{\max }, p_{\mathrm{r}}^{\max }, p_{\mathrm{s}}^{\max }\right]$ & {$[1,2,1] \mathrm{pu}$} & $c_{\mathrm{t}}^{\prime}$ & $0.7511 / \mathrm{pu}$ \\
\hline$\left[x^{\min }, x^{\max }\right]$ & {$[0,4] \mathrm{puh}$} & $c_{\mathrm{t}}^{\prime \prime}$ & $0.06931 / \mathrm{pu}^{2}$ \\
\hline$\left[\tilde{x}^{\min }, \tilde{x}^{\max }\right]$ & {$[1,3] \mathrm{puh}$} & $c_{\mathrm{t}}^{\mathrm{s}}$ & 0.3162 \\
\hline$x^{0}$ & $3 \mathrm{puh}$ & $c_{\mathrm{r}}$ & $11 / \mathrm{pu}$ \\
\hline$\left[K_{\mathrm{t}}, K_{\mathrm{s}}\right]$ & {$[1,1]$} & $\gamma$ & 0.95 \\
\hline
\end{tabular}

parameters of the units and the weights of the cost function can be found in Table [.

The controllers and the MG model in Fig. 3 were implemented in MATLAB R2019b using YALMIP R20200116 [25] and solved with Gurobi 9.0.2 on a machine with a $3.70 \mathrm{GHz}$ Intel ${ }^{\circledR} \mathrm{Xeon}^{\circledR}$ E5-1620 v2 CPU and 32 GB RAM. The computation times were reduced by using the results of the previous iterations for a warm-start of the solver. Moreover the binary variables used in the model were relaxed for stages larger than or equal to $j=4$ to speed up the solver. Note that the binary variables used to formulate the chance constraints in $\left(\mathrm{P}_{\mathrm{cc}}\right)$ were not relaxed.

The time series of load demand used in the simulations was based on measurements from a real-world islanded MG. For the PV power plant, irradiance data from [26] was employed. The time series of load and irradiance were used to train seasonal autoregressive integrated moving average (ARIMA) models [4] that are employed to forecast load and available renewable infeed for the MPC formulation. In detail, for load an $\operatorname{ARIMA}(10,0,8)(7,1,7)_{48}$ model, and for irradiance an $\operatorname{ARIMA}(6,1,2)(1,1,1)_{48}$ model were used. From both models, scenario trees of the prediction errors were constructed in a similar way as in [2], [27]. In what follows, chance constraints and the corresponding risk constraints are imposed separately for the upper and lower bounds, that is, we use $d_{j}^{\max }(x)=\min _{y \leq x_{s}^{\max }}|x-y|$, and $d_{j}^{\min }(x)=\min _{y \geq x_{s}^{\min }}|x-y|$.

\section{A. Closed-loop simulations for $\alpha=0.5$}

The results of the closed-loop simulations with the riskconstrained MPC ( $\alpha=0.5)$ over a simulation horizon of 7 d, i.e., 336 simulation steps, are shown in Fig. 4 It can be noted that during each day, PV infeed is used to charge the storage unit. During this time, the conventional unit is disabled. At the end of each day, the storage unit is discharged as the infeed from the PV power plant decreases. At night, the conventional unit is repeatedly enabled to provide power to the loads and charge the storage units. In theory, $\alpha=0.5$ allows for $50 \%$ of the predicted values to be above $\tilde{x}^{\max }=3 \mathrm{puh}$ at each stage and for $50 \%$ of the values to be below $\tilde{x}^{\text {min }}=1 \mathrm{puh}$. In the closedloop simulations, however, only 18 energy values outside the interval $\left[\tilde{x}^{\min }, \tilde{x}^{\max }\right]$ were observed with maximum distance to the interval below $0.05 \mathrm{puh}$.

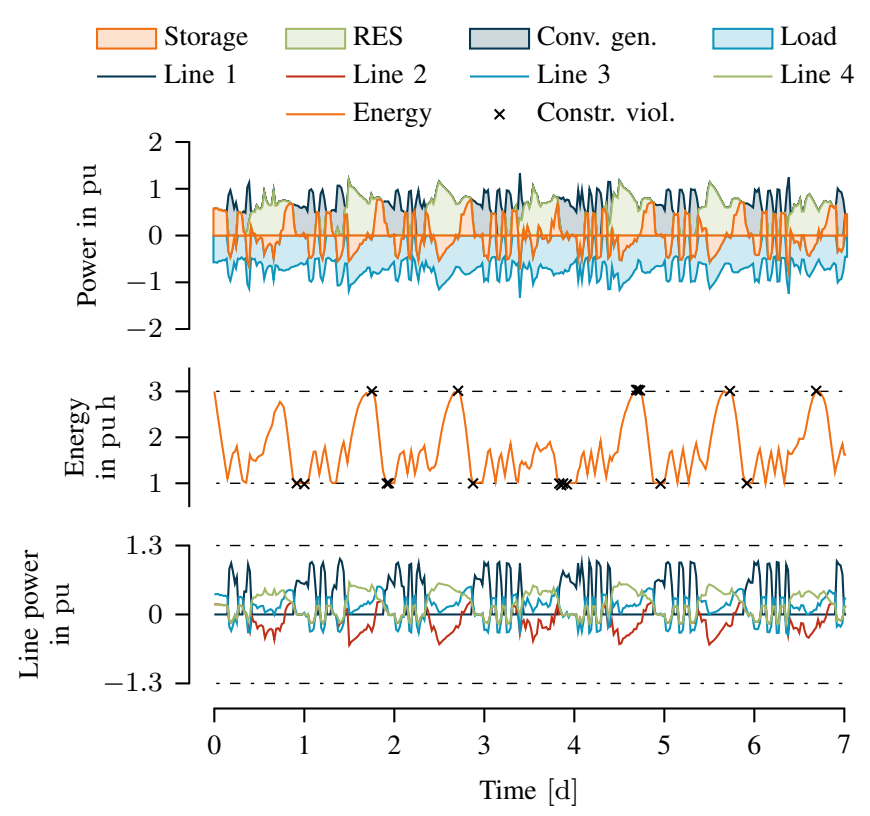

Fig. 4. Results of closed-loop simulations over one week with riskconstrained MPC at $\alpha=0.5$.

TABLE III

ACCUMULATED VALUES OF CLOSED-LOOP SIMULATION WITH SIMULATION HORIZON $K=336$.

\begin{tabular}{|c|c|c|c|c|c|c|}
\hline & \multicolumn{3}{|c|}{ Chance-constr., $\alpha=$} & \multicolumn{3}{|c|}{ Risk-constr., $\alpha=$} \\
\hline & 0.1 & 0.2 & 0.5 & 0.1 & 0.2 & 0.5 \\
\hline Avg. costs $\bar{\ell}$ & 3.35 & 3.35 & 3.3 & 3.36 & 3.36 & 3.33 \\
\hline Avg. ren. share $[\%]$ & 50 & 50 & 52.63 & 49.12 & 49.12 & 50 \\
\hline$x \notin\left[\tilde{x}^{\min }, \tilde{x}^{\max }\right]$ & 9 & 12 & 43 & 7 & 8 & 18 \\
\hline Switching actions & 15 & 15 & 39 & 15 & 15 & 48 \\
\hline Avg. solve time $[\mathrm{s}]$ & 1.05 & 2.38 & 3.62 & 0.59 & 0.69 & 1.37 \\
\hline Max. solve time [s] & 8.63 & 26.43 & 16.6 & 6.82 & 6.26 & 9.73 \\
\hline
\end{tabular}

\section{B. Comparison of closed-loop simulations for different $\alpha$}

The results of closed-loop simulations with the chanceconstraint MPC $\left(\overline{\mathrm{P}_{\mathrm{cc}}}\right)$ and the risk-constrained MPC $\left(\overline{\mathrm{P}_{\mathrm{rc}}}\right)$ for different values of $\alpha$ are shown in Table III It can be noted that with increasing $\alpha$, the average closed-loop costs

$$
\bar{\ell}=\sum_{k=1}^{336} \ell(v(k), v(k-1), p(k)),
$$

decrease. Comparing, for example, the risk-constrained MPC for $\alpha=0.11$ and $\alpha=0.5$, one can see that the average cost decreases about $1 \%$. Note that this decrease is reached solely by allowing some energy constraints violations. As noted earlier, the maximum violation of the chance constraints is less than $0.05 \mathrm{puh}$, so the decrease in price comes at the acceptable disadvantage of very small violations of $x$.

It can be seen in Table III that the number of constraint violations of the chance-constrained approach is smaller than that of the risk-constraint approach. Moreover, the average

\footnotetext{
${ }^{1}$ Note that a uniform imposition of constraints via the chance-constrained MPC with $\alpha=0$ also leads to an average cost of $\bar{\ell}=3.36$.
} 


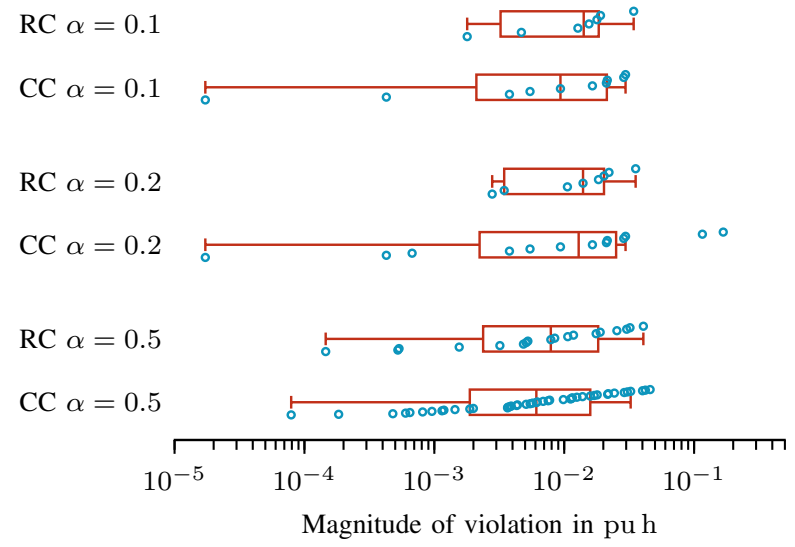

Fig. 5. Distribution of constraint violations relative to bounds, i.e., $\tilde{x}^{\min }-x(k)$ and $x(k)-\tilde{x}^{\max }$ for chance constrained $(\mathrm{CC})$ and risk constrained (RC) MPC. Note that states that did not violate any constraint were omitted.

closed-loop cost $\bar{\ell}$ of the chance-constrained MPC is slightly smaller than that of the risk-constrained approach. Both effects are based on the fact that risk constraints overapproximate chance constraints. Furthermore, the average and the maximum computing time of the solver are lower for the risk-constrained approach. Unlike chance constraints, the risk-based aporoach does not neccesitate additional binary varibles. This leads to optimization problems that can accomodate an adequate number of storage units at a reasonable computational cost.

In Fig. 5, distributions of values outside the interval $\left[\tilde{x}^{\mathrm{min}}, \tilde{x}^{\mathrm{max}}\right]$ are shown for the different MPC approaches and values of $\alpha$. It can be noted that, for the same value of $\alpha$, the risk-constrained controller typically comes with a smaller number of violations. The largest violations of all simulations occur using the chance-constrained controller. This indicates that imposing risk constraints can help to prevent extreme violations by taking their magnitude into account.

\section{CONCLUSiOnS}

This paper proposes a scenario-based stochastic model predictive control formulation with stagewise risk-based constraints on the state of charge of the storage units of a microgrid. Unlike chance constraints, risk constraints can control both the frequency and magnitude of constraint violations; they can allow infrequent and mild violations of the state-ofcharge constraints leading to a lower operating cost. Lastly, risk constraints are convex unlike chance constraints, which necessitate the introduction of binary variables; as a result, risk-constrained problems can be solved faster.

\section{REFERENCES}

[1] R. Palma-Behnke, C. Benavides, F. Lanas, B. Severino, L. Reyes, J. Llanos, and D. Sáez, "A microgrid energy management system based on the rolling horizon strategy," IEEE Trans Smart Grid, vol. 4, no. 2, pp. 996-1006, 2013.

[2] C. A. Hans, P. Sopasakis, A. Bemporad, J. Raisch, and C. ReinckeCollon, "Scenario-based model predictive operation control of islanded microgrids," in IEEE CDC, 2015.
[3] C. A. Hans, P. Sopasakis, J. Raisch, C. Reincke-Collon, and P. Patrinos, "Risk-averse model predictive operation control of islanded microgrids," IEEE Trans Contr Sys Tech, vol. 28, no. 6, pp. 21362151, 2020.

[4] G. E. Box, G. M. Jenkins, and G. C. Reinsel, Time series analysis: forecasting and control. John Wiley \& Sons, 2013.

[5] A. G. Tsikalakis and N. D. Hatziargyriou, "Centralized control for optimizing microgrids operation," in IEEE PES GM, 2011.

[6] A. Parisio, E. Rikos, and L. Glielmo, "Stochastic model predictive control for economic/environmental operation management of microgrids: An experimental case study," J Proc Contr, vol. 43, pp. 24-37, 2016.

[7] C. A. Hans, V. Nenchev, J. Raisch, and C. Reincke-Collon, "Approximate closed-loop minimax model predictive operation control of microgrids," in ECC, 2015, pp. 241-246.

[8] Y. Zhang, N. Gatsis, and G. B. Giannakis, "Robust energy management for microgrids with high-penetration renewables," IEEE Trans Sust Energy, vol. 4, no. 4, pp. 944-953, 2013.

[9] M. Gulin, J. Matuško, and M. Vašak, "Stochastic model predictive control for optimal economic operation of a residential DC microgrid," in IEEE ICIT, 2015, pp. 505-510.

[10] X. Xu, Z. Yan, M. Shahidehpour, Z. Li, M. Yan, and X. Kong, "Datadriven risk-averse two-stage optimal stochastic scheduling of energy and reserve with correlated wind power," IEEE Trans Sust Energy, vol. 11 , no. 1 , pp. 436-447, 2020.

[11] F. Oldewurtel, C. N. Jones, and M. Morari, "A tractable approximation of chance constrained stochastic MPC based on affine disturbance feedback," in IEEE CDC, 2008, pp. 4731-4736.

[12] A. Nemirovski and A. Shapiro, "Convex approximations of chance constrained programs," SIAM J Optim, vol. 17, no. 4, pp. 969-996, 2007.

[13] T. L. M. Santos, A. D. Bonzanini, T. A. N. Heirung, and A. Mesbah, "A constraint-tightening approach to nonlinear model predictive control with chance constraints for stochastic systems," in ACC, 2019, pp. 1641-1647.

[14] P. Kou, D. Liang, and L. Gao, "Stochastic energy scheduling in microgrids considering the uncertainties in both supply and demand," IEEE Systems Journal, vol. 12, no. 3, pp. 2589-2600, 2018.

[15] P. Sopasakis, M. Schuurmans, and P. Patrinos, "Risk-averse riskconstrained optimal control," in ECC, 2019, pp. 375-380.

[16] C. Hans, P. Braun, J. Raisch, L. Grüne, and C. Reincke-Collon, "Hierarchical distributed model predictive control of interconnected microgrids," IEEE Tr Sust Energy, vol. 10, no. 1, pp. 407-16, 2019.

[17] J. Schiffer, C. A. Hans, T. Kral, R. Ortega, and J. Raisch, "Modelling, analysis and experimental validation of clock drift effects in low-inertia power systems," IEEE Trans Ind Electron, vol. 64, no. 7, pp. 59425951, 2017.

[18] A. Bemporad and M. Morari, "Control of systems integrating logic, dynamics, and constraints," Automatica, vol. 35, no. 3, pp. 407-427, 1999.

[19] M. Živić Đurović, A. Milačić, and M. Kršulja, "A simplified model of quadratic cost function for thermal generators," Ann. DAAAM 2012 Proc. 23 Int. DAAAM Symp., vol. 23, no. 1, pp. 25-28, 2012.

[20] A. Ahsan, Q. Zhao, A. M. Khambadkone, and M. H. Chia, "Dynamic battery operational cost modeling for energy dispatch," in IEEE Energy Conversion Congress and Exposition, 2016.

[21] A. Shapiro, D. Dentcheva, and A. Ruszczyński, Lectures on stochastic programming: modeling and theory, 2nd ed. SIAM, 2014.

[22] A. Ahmadi-Javid, "Entropic value-at-risk: A new coherent risk measure," JOTA, vol. 155, no. 3, pp. 1105-1123, 122011.

[23] M. Schuurmans, P. Sopasakis, and P. Patrinos, "Safe learning-based control of stochastic jump linear systems: a distributionally robust approach," in IEEE CDC, 2019, pp. 6498-6503.

[24] A. Ben-Tal and A. Nemirovski, Lectures on Modern Convex Optimization. SIAM, 2001.

[25] J. Löfberg, "YALMIP: A toolbox for modeling and optimization in MATLAB," in IEEE Int Sym Comp Aid Contr Sys Des, 2004, pp. 284-289.

[26] R. Coulter, J. Kyrouac, and D. Holdridge, "ARM: Balloonborne sounding system (BBSS): Vaisala-processed winds, press., temp, and rh," 1994, accessed 14 July 2011. [Online]. Available: http://dx.doi.org/10.5439/1021460

[27] H. Heitsch and W. Römisch, "Scenario reduction algorithms in stochastic programming," Comp Optim Appl, vol. 24, no. 2-3, pp. 187206, 2003. 